Revue de l'Institut des langues et cultures

d'Europe, Amérique, Afrique, Asie et Australie

38 | 2020

Chant et nation : de la culture populaire à la culture savante

\title{
De la métraduction à la transculturation : musique et « oralité populaire » dans Concierto barroco d'Alejo
}

\section{Carpentier}

From Mistranslation to Transculturation: Music and "Popular Orality" in

Concierto barroco by Alejo Carpentier

\section{Aurélien Talbot}

\section{OpenEdition}

\section{Journals}

Édition électronique

URL : http://journals.openedition.org/ilcea/9654

DOI : 10.4000/ilcea.9654

ISSN : 2101-0609

Éditeur

UGA Éditions/Université Grenoble Alpes

\section{Édition imprimée}

ISBN : 978-2-37747-172-0

ISSN : 1639-6073

Référence électronique

Aurélien Talbot, « De la métraduction à la transculturation : musique et « oralité populaire » dans Concierto barroco d'Alejo Carpentier », ILCEA [En ligne], 38 | 2020, mis en ligne le 31 janvier 2020, consulté le 31 janvier 2020. URL : http://journals.openedition.org/ilcea/9654 ; DOI : 10.4000/ilcea. 9654

Ce document a été généré automatiquement le 31 janvier 2020

(c) ILCEA 


\title{
De la métraduction à la
} transculturation : musique et « oralité populaire » dans Concierto barroco d'Alejo Carpentier

\author{
From Mistranslation to Transculturation: Music and "Popular Orality" in \\ Concierto barroco by Alejo Carpentier
}

Aurélien Talbot

1 Le rôle structurant de la musique dans l'ensemble de l'œuvre d'Alejo Carpentier a été largement étudié ${ }^{1}$, de même que dans son roman Concierto barroco ${ }^{2}$, auquel, malgré sa brièveté, l'écrivain et musicologue cubain accordait une importance particulière ${ }^{3}$. Dans le cadre du présent article, il ne s'agira toutefois pas de poursuivre l'exploration des liens intriqués entre écriture et musique chez Carpentier, mais de proposer une lecture du roman fondée sur l'hypothèse que la coexistence et l'articulation d'une pluralité de visions de la musique, représentées par différents épisodes musicaux et personnages musiciens, ouvrent des aperçus sur le phénomène de la traduction.

2 Il nous est en effet apparu que ce roman, de même que d'autres œuvres de la seconde moitié $\mathrm{du} \mathrm{xx}^{\mathrm{e}}$ siècle $^{4}$, était à même de susciter plusieurs types de réflexions traductologiques, concernant la traduction au sens restreint de traduction entre les langues (comment traduire les différents registres, les expressions vernaculaires, les phrases longues et chargées de Carpentier en français?), mais aussi au sens large, "métaphorique », lorsque ce mot désigne non seulement «le "passage" interlangues d'un texte, mais - autour de ce premier "passage" - toute une série d'autres "passages" " (Berman, 1999: 20-21) et, dans le cas qui nous occupe, le passage de cultures musicales de l'Europe à l'Amérique et retour.

3 Nous nous attacherons ainsi tout d'abord à dégager dans Concierto barroco plusieurs motifs musicaux évoquant les procédures de passage, de translation et de traduction au sens large, avant d'esquisser un rapport entre ces motifs - relus à la lumière de 
certaines remarques de l'écrivain cubain sur le roman latino-américain - et quelques textes du traductologue Antoine Berman dont la réflexion a été fortement marquée, comme nous essaierons de le montrer, par son expérience de traducteur d'œuvres littéraires latino-américaines.

\section{1. "Mojiganga mexicana ${ }^{5}$ » et " jam session » : « inventer une histoire »}

Concierto barroco a pour noyau un opéra de Vivaldi intitulé Motezuma. L'idée du roman serait en effet venue à Carpentier après qu'un compositeur italien, Francesco Malipiero, lui a fait prendre connaissance, dès 1936, de l'existence de cet opéra, créé par Vivaldi en 1733 à Venise, sur un livret d'Alvise (Luigi) [Girolamo ?] Giusti, opéra qui a pour sujet la Conquête du Mexique (Chao, 1998: 70). Bien des années après, le musicologue français Roland de Candé, spécialiste de Vivaldi, remettra Carpentier (1998 : 99) sur la piste de cet opéra ${ }^{6}$.

Or Carpentier semble avant tout intéressé par cette œuvre du fait qu'il s'agit du « premier opéra sérieux inspiré par la Conquête du Mexique » (1976 : 117). Le relevé des précédents et, plus encore, des livrets ultérieurs d'opéras ayant pris pour sujet la Conquête du Mexique, a donné lieu à des recherches sur lesquelles nous ne reviendrons pas (Vásquez, 1991: 130-132; Montoya Campuzano 2001 : 296-297). Dans le cadre qui est le nôtre, nous voudrions uniquement faire observer qu'un double mouvement est perceptible au septième chapitre du roman qui a pour contexte la répétition générale de cet opéra à Venise en 1733, sous le regard stupéfait du personnage principal, un Mexicain en voyage en Europe, continent d'où étaient originaires ses grands-parents espagnols.

Suivant un premier mouvement, Carpentier souligne le peu de rigueur historique du livret et les modalités suivant lesquelles la Conquête du Mexique y est constamment relue en fonction des coordonnées du théâtre antique européen, le personnage de Montezuma se coulant notamment dans le rôle de Xerxès (Carpentier, 1998: 65), son épouse dans celui de Sémiramis (ibid. : 68) et sa fille dans celui d'Iphigénie (ibid. : 71). La lagune de Mexico-Tenochtitlán est par ailleurs assimilée à la lagune de Venise (ibid. : 65 et 67).

7 Le deuxième mouvement observable dans le texte de Carpentier concerne au contraire la précision et le soin érudits avec lesquels Carpentier traite les détails historiques qui entourent cet opéra. Concernant par exemple la distribution: les noms mentionnés dans Concierto barroco (Carpentier, 1998 : 66) sont ceux-là mêmes des chanteuses et des chanteurs qui ont joué cet opéra en 1733 (Müller-Bergh, 1975: 461-462 et Vickers, 2006 : 51). Pareillement, concernant les décors : les descriptions des scènes de l'opéra dans le roman suivent presque à la lettre les didascalies du livret (Mimoso-Ruiz, 1980 : 129-133). De façon générale, le portrait de Vivaldi et la fresque de Venise au XVIII ${ }^{\mathrm{e}}$ siècle s'appuient sur un faisceau de sources fiables (Müller-Bergh, 1975 : 456-462 et MimosoRuiz, 1980 : 122-126) qui ne laissent rien au hasard.

De sorte que ce double mouvement en sens contraire du texte de Carpentier peut conduire à deux observations. D'une part, on peut y déceler une méthode d'écriture propre à Carpentier qui a pu un jour déclarer: "Je suis absolument incapable d'inventer une histoire. Tout ce que j'écris relève d'un montage de choses vécues, 
observées, de souvenirs, regroupés par la suite en un ensemble cohérent ${ }^{7}$. Or il nous semble qu'il est possible de prendre cette affirmation à la lettre et non comme une déclaration rhétorique. Carpentier, à cet égard, s'inscrirait dans un large mouvement par lequel tout un pan de l'écriture bascule progressivement dans la description et l'agencement de pièces (qu'il s'agisse de blocs de mémoire ou de faits rapportés), la priorité étant accordée à la facture du style et au discours indirect ${ }^{8}$.

D'autre part, et ce sera notre deuxième remarque concernant ce double mouvement antagoniste qui caractérise la mise en intrigue de l'opéra de Vivaldi dans Concierto barroco, tout se passe comme si Carpentier mettait au jour des procédures de traduction ou de translation là où le livret de l'opéra s'efforçait de les cacher ou de les gommer, ou plutôt comme si Carpentier, par l'ironie qu'il distille, les remarques de ses personnages et plusieurs autres procédés d'écriture parmi lesquels la citation et le collage (MimosoRuiz, 1980 : 129-133 et Müller-Bergh: 457), mettait l'accent sur les phénomènes d'annexion ou de métraduction dans le livret d'opéra qui obéit à une esthétique de la réception accordant la priorité au divertissement du spectateur' ${ }^{9}$. Cette ironie de Carpentier insiste sur le caractère mythologisant et les déformations interprétatives propres à l'opéra Motezuma.

10 À cette étape de notre développement, il faut rappeler que Carpentier s'est lui-même essayé au récit de la Conquête du Mexique dans une pièce de théâtre écrite en 1956 en français (et peut-être n'est-ce pas un hasard), contrairement au reste de son œuvre littéraire écrite en espagnol (Moulin Civil, 2012 : 96-97). Cette pièce de théâtre, intitulée L'apprentie sorcière (Carpentier, 1990), choisit de traiter la Conquête du point de vue du personnage historique de Malintzin, également appelée Marina ou Malinche, qui fut l'interprète et l'amante du conquistador Hernán Cortés ${ }^{10}$. Or, dans Concierto barroco, le riche Mexicain en visite en Europe fait précisément remarquer à Vivaldi que le personnage de « Doña Marina » (Carpentier, 1998: 75) est absent de son opéra. Dans la pièce de théâtre de Carpentier, Malintzin, Marina ou Malinche, incarne le point de rencontre où s'inversent les perspectives, le foyer de la vision où s'entrechoquent "légende dorée " et "légende noire", la perspective des vainqueurs et celle des vaincus de la Conquête. Carpentier montre ainsi en une même pièce les deux profils de cette figure historique : le profil de Doña Marina en tant que première Indienne sauvée des affres de l'idolâtrie et le profil de la Malinche en tant que première traitresse du peuple mexicain.

11 Dans ce cadre, qui peut évoquer, comme Moulin Civil (2012: 99) en formule l'hypothèse, une réponse de Carpentier à Octavio Paz (2004: 94-97) à propos de la controverse suscitée par cette figure de la Malinche, nous voudrions souligner que la logique historique et la logique de la traduction apparaissent solidaires ${ }^{11}$. Ainsi, s'il est vrai que, dans Concierto barroco, comme le suggèrent plusieurs commentateurs (Acosta, 1981 : 97-98; Vásquez 1991: 133 ; Mimoso-Ruiz, 1980 : 135 ; Martín Ghioldi, 2007 : 115-118), l'opéra Motezuma permet au personnage du Maître, dans son Odyssée qui va de Coyoacán à Venise, dans son Grand Tour de l'Europe, de reconnaître son identité américaine, il serait alors possible de concevoir que ce bref roman, qui se termine par diverses allusions au Messie, à la fin des temps et au Jour du Jugement (Carpentier, 1998: 81-90), propose, en son fond, une figure bien particulière d'eschatologie, une forme, selon l'expression d'Éric Dayre (2017:69) d'« eschatologie disproportionnée » passant par un dialogue "ironique, impur, heurté, et en quelque manière ruiné " (Dayre, 2009 : 9) débouchant sur « une certaine forme de la fin [...] et du remplacement 
de la transition douce entre les voix par des discontinuités, des formes abruptes et des hétérogénéités perceptibles » (ibid. : 10).

En effet, la mise en parallèle de la logique historique et de la logique de la traduction dont il nous semble que Carpentier fait ressortir l'importance dans son récit de la Conquête du Mexique, continue à être approfondie dans Concierto barroco à travers le personnage de Filomeno, un Cubain noir qui fait office de valet du Maitre mexicain en Europe. Or dans une scène qui occupe tout le cinquième chapitre du roman, on assiste à ce qui est qualifié de "mermelada», marmelade, confiture ou «jam session» musicale (Carpentier, 1998: 59), réunissant un orchestre d'orphelines musiciennes, Vivaldi au violon, Scarlatti au clavecin, Haendel à l'orgue, et Filomeno qui improvise à l'aide de percussions de fortune pendant 32 mesures avant d'entraîner tout le monde dans le "Chant de la couleuvre ", un chant traditionnellement interprété lors de la fête des Rois à Cuba (Carpentier, 1972 : 291-292).

De même qu'une analogie avait été effectuée entre la lagune de Mexico-Tenochtitlán et celle de Venise dans le Motezuma de Vivaldi, là aussi, tout part d'une analogie : un tableau représentant Adam, Ève et le serpent évoque pour Filomeno le chant traditionnel de la couleuvre ${ }^{12}$. Mais là où, dans l'opéra Motezuma, de l'analogie découlait un processus d'annexion ou de métraduction, selon un cercle herméneutique fermé où la Conquête du Mexique était interprétée en fonction de la provenance gréco-romaine et de la destination de l'évangélisation universelle; dans ce cas, l'analogie entre le serpent du jardin d'Éden et le chant de la couleuvre cubain permet à Carpentier de mettre en scène ce que nous appelions à l'instant une forme de dialogue «ironique, impur, heurté, et en quelque manière ruiné " ou encore une manière d'" ouvrir les fins » (Dayre, $2017:$ 87-90), c'est-à-dire de rouvrir le cercle herméneutique. Et rouvrir le cercle herméneutique correspond peut-être simplement à ce qu'on appelle en outre « inventer une histoire».

14 Il faut d'ailleurs souligner l'importance de ce personnage de Filomeno. Car si le Motezuma de Vivaldi est bien à la source de l'idée du roman Concierto barroco, il convient de noter que Filomeno est préfiguré dans plusieurs écrits antérieurs de Carpentier (Montoya Campuzano 2001: 64-65). Ce personnage, qui semble hanter Carpentier, se présente dans Concierto barroco comme l'arrière-petit-fils de Salvador Golomón, le héros du poème cubain Espejo de paciencia datant de 1608, dans lequel, comme l'a plusieurs fois fait remarquer l'auteur (Carpentier, 1998: 27 ou 1984:211), une scène de fête musicale est décrite mêlant des instruments européens et indigènes. Or, Filomeno, dans Concierto barroco, ne paraît pas jouer seulement le rôle d'ambassadeur de la musique afro-cubaine, mais aussi, plus profondément, de représentant de ce paramètre fondamental de l'Amérique latine pour Carpentier qu'est la "créolité » (Carpentier, 1984 : 211-212). À ce titre, Filomeno, sur qui se termine le roman alors qu'il entre dans une salle de concert où doit jouer le trompettiste Louis Armstrong, après une nouvelle analogie entre le Messie de Haendel et les spirituals noirs américains (Carpentier, 1998 : 89) - Filomeno est l'un des opérateurs de la traduction et, de manière concomitante, de cette articulation entre logique historique et logique de la traduction qui a permis d'évoquer ci-dessus une "eschatologie disproportionnée ", c'est-à-dire la possibilité d'un «demi-cercle» herméneutique ouvert et improvisé (Dayre, 2017: 90-93), interrogeant les concepts de créolité, mais aussi de métissage ou de "transculturation" que l'on doit à Fernando Ortiz, compagnon de jeunesse de Carpentier (1984:275-303) au sein du Grupo de los minoristas ${ }^{13}$. 
15 Avant de passer à l'examen des modalités selon lesquelles la langue espagnole de Carpentier s'ouvre pour accueillir cette créolité, nous voudrions terminer cet aperçu très succinct des visions de la musique dans Concierto barroco en relevant un exemple de traduction, au sens restreint cette fois, que l'on y trouve. Filomeno, alors qu'il entraîne Vivaldi, Haendel, Scarlatti et les orphelines de l'Ospedale della Pietá dans la comparsa cubaine du «Chant de la couleuvre » prononce une jitanjáfora, c'est-à-dire cette « sorte de poésie sonore où des mots inventés pour leurs qualités sonores alternent avec des mots existants ou les imitent» (Greene \& Cushman, 2012: 763; notre traduction), procédé qui a été en particulier employé par des poètes cubains ${ }^{14}$. Il s'agit de la suite onomatopéique "Ca-la-ba-són, són, són » qui devient, dans la bouche de Vivaldi et des autres musiciens européens «Kábala-sum-sum-sum ». Or cette traduction privilégie les signifiants - le latin "Kábala-sum-sum-sum » mimant phonétiquement "Ca-la-ba-són, són, són »- sans abandonner pour autant le sens, si le geste musical peut en avoir un, étant donné que la cabale peut rappeler, comme science occulte, les rituels des " diablitos » noirs du jour des Rois à Cuba (Carpentier, 1972 : 301). Carpentier propose ainsi, à travers cette traduction homophonique ou cette "traducson » selon le mot valise créé par Nardipher et repris par Genette (1982: 50), un trait d'esprit et de traduction.

\section{Créolité et polymétissage : un « défi » pour la traduction}

16 Après avoir brièvement relevé, dans la trame de Concierto barroco, quelques motifs liés à des procédures de traduction, de translation ou de transculturation, et avoir cherché à indiquer la réflexion qui pourrait en découler du point de vue de l'articulation entre logique historique et logique de la traduction, nous voudrions maintenant approfondir l'hypothèse que ce bref roman peut contribuer à approcher le phénomène de la traduction en confrontant, comme annoncé, certains écrits du traducteur et traductologue Antoine Berman aux réflexions de Carpentier sur le roman.

17 À cet égard, on peut rappeler que Berman était traducteur de romans latinoaméricains, qu'il a notamment traduit l'écrivain paraguayen Augusto Roa Bastos (1977) et l'écrivain argentin Roberto Arlt (1981 et 1984) et que cette expérience semble avoir exercé une influence fondatrice sur sa réflexion traductologique, comme en attestent quelques-uns de ses articles un peu oubliés (Berman, 1979; 1980 ; 1982; 1985) et plusieurs passages de ses ouvrages ultérieurs plus connus (Berman, 1995a: 38-39; $1995 b$ : 84-85 et $175-184 ; 1999: 14$ ou $61-62$ ou $66 ; 2008: 60-63)$.

Par ailleurs, il arrive à Berman de mentionner Carpentier. Ainsi, dans une note de Pour une critique des traductions: John Donne, Berman (1995b:46) fait référence à une thèse (Cordonnier, 1989) dans laquelle est analysée (ibid.: 353-406) la traduction française d'un roman de Carpentier, El arpa y la sombra, traduction réalisée, comme celle de Concierto barroco, par René L. F. Durand (de son vrai nom René Marc Ducaud) qui était le «traducteur attitré » (Berman, 1995b : 46) de Carpentier ${ }^{15}$. En outre, dans l'article « La voix des sans-voix : Arguedas, García Márquez, Scorza, Roa Bastos », Berman (1980 : 4) évoque le roman Los pasos perdidos de Carpentier et revient sur les réflexions de l'écrivain cubain concernant le roman latino-américain pour les considérer à la lumière des spécificités des romanciers qu'il choisit de mettre en avant. 

et 1995a: 39) que pose la traduction des œuvres littéraires latino-américaines en français et associe ce « défi » à ce qu'il désigne comme un "desencuentro linguistique et culturel » $(1982: 39)$. Or un tel « desencuentro linguistique et culturel » paraît faire écho, dans le cadre de la réflexion bermanienne en germe à ce moment-là, au "desencuentro " originel entre les Espagnols et les Aztèques, qualifié d'« entrechoc [...] [qui] mènera à un poly-métissage généralisé » (Berman, $1980: 4)$, et dont la figure de l'interprète et amante de Cortés, Doña Marina, demeure emblématique. Par là même, nous retrouvons l'entrelacement des logiques historique et de la traduction. En effet, à la suite de "l'entrechoc» ou du "desencuentro", la question qui se pose, d'un point de vue éthique, aussi bien au niveau culturel (historique) que linguistique (traductionnel), consiste à déterminer comment rendre justice au " poly-métissage ", à la "violence du métissage » (Berman, 1995a: 16) qui en résulte? Telle est fondamentalement, pour Berman, l'origine du «défi » posé par les œuvres littéraires latino-américaines au traducteur français.

Or sur ce point, la réflexion bermanienne nous semble entrer en résonance avec certaines observations de Carpentier. Car Berman comme Carpentier, ainsi que nous allons chercher à le montrer, critiquent la langue française conçue comme un ensemble de normes classiques et rigides et mettent en question, plus généralement, la vigueur des langues et des littératures européennes que les romanciers latino-américains (dont ils comparent tous deux l'activité à celle des auteurs du xvi siècle européen) seraient susceptibles de revivifier ${ }^{16}$.

(at bermanien est en effet sans appel: en 1979, il affirme que «notre roman est en général, actuellement, médiocre [...] nos recherches formelles [...] ne dépassent guère, sauf exceptions, le cadre d'une expérimentation exsangue » (Berman, 1979 : 163). Après quoi il ajoute que «García Márquez, Lezama Lima, Carpentier, Roa Bastos, etc., ont pour pairs Broch, Joyce, Musil, Mann, Kafka, Virginia Woolf, mais aucun écrivain européen actuel» (ibid. : 164). Quelques années plus tard, en 1984, dans L'épreuve de l'étranger, Berman poursuit sa réflexion et observe que "comme les auteurs du xvi siècle européen, Roa Bastos, Guimarães Rosa, J.-M. Arguedas - pour ne citer que les plus grands -- écrivent à partir d'une tradition orale et populaire » (1995a : 38). Si bien que le « défi » que rencontre le traducteur français face aux œuvres littéraires latinoaméricaines (« défi » qui, encore une fois, ne serait, dans ce cadre, qu'une conséquence de plus du "desencuentro " originel) apparaît concrètement lié à une "oralité ", caractéristique qui serait perdue par la littérature française et européenne depuis le tournant du classicisme :

Le travail à accomplir sur le français moderne pour le rendre capable d'accueillir authentiquement, c'est-à-dire sans ethnocentrisme, ce domaine littéraire montre bien qu'il s'agit, dans et par la traduction, de participer à ce mouvement de décentrement et de changement dont notre littérature (notre culture) a besoin si elle veut retrouver une figure et une expérience d'elle-même qu'elle a en partie perdues (pas totalement, bien sûr !) depuis le classicisme. (Berman, 1995a : 39)

La traduction de romans latino-américains semble ainsi fournir à Berman l'occasion de retravailler la notion d'oralité qu'il emprunte à Meschonnic ${ }^{17}$. Berman parle en effet de «l'oralité des œuvres latino-américaines" (1982: 40), de «textes enracinés dans la culture orale » (1995a : 39) ou encore du « rapport complexe qui lie la littérature latinoaméricaine au langage oral populaire " (1979: 165). Face à une telle "oralité ", compte tenu des «tendances antivernaculaires de notre langue littéraire » (Berman, 
1982 : 40), « il y a péril à la traduction d'un resserrement rationalisant » (ibid.) et la liste des célèbres "tendances déformantes" que l'on trouve dans La traduction et la lettre (Berman, 1999 : 52-68) ayant donné lieu à tant de commentaires, sont une première fois partiellement recensées par Berman (1982: 40-42) dans le cadre d'une réflexion sur la littérature latino-américaine.

Ce constat concernant le « défi » posé par la traduction des œuvres littéraires latinoaméricaines en français est partagé et développé par Inês Oseki-Dépré (2007 : 37). Il est possible de le mettre en perspective ${ }^{18}$, mais ce qui retiendra ici notre attention est le parallèle qui peut être établi avec certaines déclarations de Carpentier. En effet, comme on le sait, Carpentier entretient un rapport intense au français, notamment du fait de ses origines (Chao, 1998: 26), et le choix qu'il a fait d'écrire ses œuvres en espagnol répond, selon ses déclarations, à une volonté explicite ${ }^{19}$. Ainsi Carpentier affirme-t-il qu' "[il] a préféré l'espagnol » car d'après lui «il s'agit d'une langue magnifique, à la souplesse, à la richesse et aux ressources littéraires incomparables " $(1984: 133)^{20}$. Il ajoute qu'avec l'espagnol, contrairement au français, il est possible de :

[...] faire éclater la langue si nécessaire. On invente un mot en espagnol, on substantivise un verbe, et la langue admet si bien ce type de jeux que tout le monde comprend et personne ne trouve une phrase obscure lorsqu'elle contient un verbe bizarre inventé par un écrivain. Avec l'espagnol, on peut tout faire. Ce qui n'est pas le cas avec le français. (Ibid.)

Carpentier distingue ainsi l'espagnol du français car il rechercherait cette souplesse et cette richesse incomparables, tout ce que Berman considère comme des traits distinctifs de «l'oralité populaire» (2012: 221) ou encore de «l'essence dialectale de la langue » (2008: 181), traits qui se heurteraient, d'après lui, à « la tendance historique de notre langue littéraire [qui] a consisté depuis le classicisme à bannir tout polyfacétisme et tout lien avec le vernaculaire » $(1982: 39)^{21}$.

24 À cet égard, il convient de noter que Carpentier distingue non seulement l'espagnol du français mais aussi l'espagnol d'Espagne de l'espagnol d'Amérique latine :

Si j'ai écrit mon œuvre littéraire en espagnol, ce n'est pas seulement parce que je considère le français comme une langue très rigide, mais aussi parce que l'espagnol est l'une des langues les plus riches du monde, car elle est constituée du castillan, de l'espagnol d'aujourd'hui, mais aussi de l'espagnol d'Amérique latine, lequel est d'une diversité extraordinaire, comme en attestent certains romans sudaméricains. Moi, le Suprême en constitue un bon exemple. (Chao, 1998: 27 ; notre traduction $^{22}$

De ce point de vue ${ }^{23}$, il semblerait que si l'espagnol est, pour Carpentier, plus souple que le français, ce serait principalement en raison de ses variantes latino-américaines.

Par ailleurs, comme on le sait, l'un des projets dans lesquels souhaite s'inscrire Carpentier est précisément l'invention d'une prose pour l'Amérique latine, avec pour corollaire l'invention d'un roman latino-américain. Il s'agit de :

[...] créer une langue qui se différenciera chaque jour davantage de la langue d'Azorín, de la langue des Pérez de Ayala, pour atteindre un mode d'expression qui soit le nôtre. Je sais combien cette entreprise est difficile; une grande responsabilité pèse sur nos épaules, à nous, romanciers d'aujourd'hui, qui manions, recréons et reconstituons une langue qui puisse être à nous. (Carpentier, 1984 : 142)

Comment faire, par conséquent, en traduction française ? Comment relever le « défi » spécifique que poseraient, dans ce contexte, les œuvres littéraires latino-américaines?

À ce sujet, Berman (1982: 42-43) propose une série de remarques, fondées sur son expérience de traducteur, et susceptibles de fournir des pistes concrètes. Or, dans ce 
cadre, le nom d'un écrivain déjà mentionné revient à plusieurs reprises sous sa plume et, ce qui nous intéresse tout particulièrement, sous celle de Carpentier également, comme contre-exemple des tendances classicisantes de la langue littéraire française ${ }^{24}$. En effet, lorsque Carpentier évoque une langue française souple et riche qui correspond à son idée du baroque, il mentionne Rabelais qu'il qualifie d'« inventeur de mots, d'enrichisseur de la langue, qui se permettait tous les luxes, car lorsque les verbes lui manquaient, il les inventait et lorsqu'il ne disposait pas d'adverbes, il les inventait aussi » $(1984: 110)^{25}$. Pour sa part, Berman fait la remarque suivante entre autres nombreuses remarques où il invoque Rabelais ${ }^{26}$ :

Dans le cas d'une œuvre latino-américaine, on est presque toujours en présence d'un système lexical déterminé et d'une prolifération linguistique renvoyant à un enracinement oral. Car l'oralité emploie à la fois des mots-clefs et « beaucoup » de mots : pensons à Rabelais ! $(1982: 43)$

Or le réseau qui permet ainsi de faire communiquer les textes de Carpentier et de Berman passe sans doute par une lecture commune de Bakhtine et notamment de son ouvrage consacré à Rabelais (Bakhtine, 1985) ${ }^{27}$. De sorte que pour Berman, traduire des œuvres latino-américaines en français, implique non pas, contrairement à ce que préconisait Pannwitz pour l'allemand ${ }^{28}$, «de latino-américaniser le français» mais de "mobiliser [dans la langue française] des potentialités linguistiques et culturelles homologues " (1982: 42) ou encore, comme déjà mentionné, pour "notre littérature (notre culture) » de "retrouver une figure et une expérience d'elle-même qu'elle a en partie perdues " (1995a : 39) ${ }^{29}$. Ce qui veut dire "chercher les points "lâches" » de la langue française car " ces points "lâches" existent, tout comme existent en espagnol de tels points pour accueillir des structures syntactiques quechua ou guarani » (Berman, 1982: 42). Dans «L'avant-propos» de la traduction des Sept fous de Roberto Arlt, Isabelle et Antoine Berman affirment qu'il leur a fallu «chercher en français [...] les mailles les plus lâches, donc les plus ouvertes du filet [de la langue française]» (2010:18). Ailleurs, pour expliciter la même idée, Berman mentionne l'expression, empruntée au traducteur Pierre Leyris, de "passages dérobés» dans la langue (1995b : 222).

Mais en quoi ces remarques sur la traduction au sens restreint des œuvres littéraires latino-américaines pourraient-elles contribuer à approcher, plus largement, le phénomène de la traduction? En fait, Berman ne cessera d'approfondir, semble-t-il, à partir de ce noyau de réflexions sur les œuvres littéraires latino-américaines et leurs traductions en français, la notion d'« oralité » en tant que «non point le "parlé" par rapport à l'"écrit", mais cet ensemble de formes qui naissent dans les voix, et que l'écriture, de Rabelais au Joyce de Finnegans Wake ou à Guimarães Rosa, peut accueillir en son sein, et se remémorer comme sa source et son origine » (2012:72). Il étendra et précisera progressivement la portée de cette notion ${ }^{30}$ pour en faire, en dernier ressort, une caractéristique de la copia, un concept qu'il forge pour dégager ce qu'est la traduction $^{31}$ :

À l'oralité populaire correspond une prose abondante, proliférante, longue, chargée d'incidentes, de ruptures, de digressions, une prose souvent lourde, obscure, mélangée, formellement négligée et non maîtrisée, riche en images et en mots iconiques. L'oralité populaire, de fait, est dominée par la copia, sous l'aspect lexical (beaucoup de mots d'horizons divers), métaphorique (beaucoup d'images, de tropes, de figures) et syntaxique (beaucoup de phrases, de formes de phrases, et de phrases longues). (2012: 221) ${ }^{32}$ 
Or il nous parait décisif que la notion d'oralité, que Berman emprunte dans un premier temps à Meschonnic et qui donnera lieu, en fin de compte, à une réflexion conceptuelle autour de la copia, passe dans l'intervalle par une refonte complète dans le creuset de l'expérience traductive de romans latino-américains. Il est en effet possible de supposer que la traduction de romans latino-américains a fourni à Berman la matrice de ce qu'il entendra plus tard par «écriture-de-traduction ${ }^{33}$ ». Dans ce contexte, le « défi » que posent au traducteur français les œuvres littéraires latino-américaines, dont l'origine est liée pour Berman (1982:39), comme nous l'avons vu, à un «desencuentro linguistique et culturel », peut donner lieu, s'il est relevé, à une prose de traduction "polylangagière » et "polymétissée » (1999: 51 et 1979: 64). Quand Berman écrit à propos d'Arguedas qu'" on a l'impression de lire un texte secrètement bilingue, où le quechua travaille sous l'espagnol » $(1980: 4)$ ou bien lorsqu'il pointe chez Roa Bastos "une déroutante multiplicité de niveaux: guarani, espagnol populaire guaranisé, espagnol "officiel", espagnol du XVIII', $\mathrm{XIX}^{\mathrm{e}}$ et $\mathrm{xx}^{\mathrm{e}}$ siècles, langages sectoriels, français, portugais, anglais, latin, grec» (ibid.), il vise une «hétéroglossie» (1999:66) aux accents bakhtiniens qui fera pour lui tout l'intérêt d'une "écriture-de-traduction ${ }^{34}$ ». Sur ce point, il rejoint, nous semble-t-il, certaines remarques de Carpentier dont le bref roman Concierto barroco présente une source féconde de réflexions sur la gamme étendue qui peut aller de la métraduction à la transculturation en passant par la traduction et la translation.

\section{Le retournement carnavalesque des voix}

31 Au terme de ce parcours, une interrogation commune, déjà partiellement formulée, pourrait permettre de récapituler notre lecture croisée de Concierto barroco, de certaines remarques de Carpentier sur le roman latino-américain et de plusieurs écrits précoces de Berman : comment rendre justice au niveau aussi bien culturel (historique) que linguistique (traductionnel) à ce que Carpentier appelle la « créolité » et Berman le " polymétissage » issus, malgré tout, d'un « desencuentro » dont la scène originelle serait fournie par le face-à-face entre Cortés et Montezuma avec, entre les deux, le personnage ambigu de Malintzin-Doña Marina-Malinche ${ }^{35}$ ?

Comme nous avons voulu le montrer, dans Concierto barroco, cette interrogation est notamment abordée en manifestant toute une gamme de procédures qui vont de la métraduction (le livret du Motezuma de Vivaldi) à la transculturation (le " concerto grosso » qui devient « jam session » avec Filomeno). Nous avons également voulu insister sur le fait que les célèbres «tendances déformantes » identifiées par Berman dans son «analytique de la traduction» (qui ouvre sur une «éthique de la traduction» dans Berman, 1999 : 49-78) sont présentées en premier lieu dans un article consacré à « La traduction des œuvres latino-américaines » (1982).

Dans les deux cas, si la visée éthique n'est jamais abandonnée, aucune illusion ne semble néanmoins aveugler les deux auteurs quant à la finesse des nuances qui vont de la métraduction à la transculturation. Berman précisera ainsi qu' « aucun traducteur ni aucun théoricien " n'échappe à la "figure essentielle et régnante de la traduction occidentale " (1999: 26), c'est-à-dire annexionniste et ethnocentrique. De même, il ne faut pas s'étonner que le critique Mimoso-Ruiz ait pu relever, d'une part, une « attitude ambiguë » (1980: 128) de Carpentier à l'égard du Motezuma de Vivaldi dans Concierto barroco et, d'autre part, le caractère de "conquête à rebours de l'espace européen " 
(ibid. : 135) que revêt le périple du Maître et de Filomeno. Le legs de Bakhtine consiste aussi, semble-t-il, dans cette conscience historique d'un possible retournement carnavalesque des voix ${ }^{36}$.

\section{BIBLIOGRAPHIE}

Acosta Leonardo (1981), Música y épica en la novela de Alejo Carpentier, La Havane : Letras Cubanas.

ARLT Roberto (1981), Les sept fous (I. et A. Berman, trad.), Paris : Belfond.

ARLT Roberto (1984), Le jouet enragé (A. Berman, trad.), Grenoble : Presses universitaires de Grenoble.

BAKHTINE Mikhaïl (1985), L'œuvre de François Rabelais et la culture populaire au Moyen Âge et sous la Renaissance [1970] (A. Robel, trad.), Paris : Gallimard.

BENJAMIN Walter (2000), « La tâche du traducteur » [1923], CEuvres I (M. de Gandillac et R. Rochlitz, trad.), Paris : Gallimard, 244-262.

BERMAN Antoine (1979), « L'édition française de América latina en su literatura », Cultures, 6(2), Paris : Les Presses de l'Unesco et la Baconnière, 163-168.

BERMAN Antoine (1980), « La voix des sans-voix : Arguedas, Garcia-Marquez, Scorza, Roa-Bastos », Canal, 39, Paris : Communication, Arts et Cultures, 4-5.

BERMAN Antoine (1982), « La traduction des œuvres latino-américaines », Lendemains, 27, Cologne : Pahl-Rugenstein, 39-44.

BERMAN Antoine (1985), «La traduction des œuvres latino-américaines en France », Multilingua, 4(4), Amsterdam, Berlin, New York : Mouton de Gruyter, 208-209.

BERMAN Antoine (1995a), L'épreuve de l'étranger. Culture et traduction dans l'Allemagne romantique [1984], Paris : Gallimard.

BERMAN Antoine (1995b), Pour une critique des traductions : John Donne, Paris : Gallimard.

BERMAN Antoine (1999), La traduction et la lettre ou l'auberge du lointain [1985], Paris : Le Seuil.

BERMAN Antoine (2012), Jacques Amyot, traducteur français. Essais sur les origines de la traduction en France, Paris : Belin.

CARPENTIER Alejo (1972), La música en Cuba [1946], Mexico : Fondo de Cultura Económica.

CARPENTIER Alejo (1976), Concert baroque (R. L. F. Durand, trad.), Paris : Gallimard.

CARPENTIER Alejo (1984), Ensayos, La Havane : Letras Cubanas.

CARPENTIER Alejo (1990), La aprendiz de bruja [1956], Obras completas de Alejo Carpentier IV, Mexico, Madrid, Bogota : Siglo Veintiuno, 25-143.

CARPENTIER Alejo (1991), Concierto barroco / Concert baroque (R. L. F. Durand, trad.) Paris : Gallimard. CARPENTIER Alejo (1998), Concierto barroco [1974], Madrid : Alianza Editorial. 
CHAO Ramón (1998), Conversaciones con Alejo Carpentier, Madrid : Alianza Editorial.

CORDONNIER Jean-Louis (1989), L'homme décentré, culture et traduction, traduction et culture (thèse de doctorat), Université de Franche-Comté, Besançon.

DAYRE Éric (2009), L'absolu comparé : essai sur une séquence moderne. Coleridge, De Quincey, Baudelaire, Rimbaud, Paris : Hermann.

DAYRE Éric (2017), « Tâche du traducteur, Tâche de l'historien, Walter Benjamin », É. Dayre \&

M. Panter (dir.), Traduction et événement, Paris : Hermann, 57-93.

DEGUY Michel (1973), Tombeau de Du Bellay, Paris : Gallimard.

De Launay Marc (2006), Qu'est-ce que traduire?, Paris : Vrin.

DELISLE Jean \& WOODSWORTH Judith (1995), Les traducteurs dans l'histoire, Ottawa : Les presses de l'université d'Ottawa ; Paris : Éditions Unesco.

GENETTE Gérard (1982), Palimpsestes. La littérature au second degré, Paris : Le Seuil.

GREENE Roland \& Cushman Stephen (dir.) (2012), The Princeton Encyclopedia of Poetry and Poetics, Princeton : Princeton University Press.

MALINGRET Laurence (2002), Stratégies de traduction : les lettres hispaniques en langue française, Arras : Artois Presses Universités.

MARTíN GHIOLDI Ernesto (2007), «Situaciones de transculturación a través de expresiones artísticas en Concierto Barroco de Alejo Carpentier», Virajes, Revista de antropología y sociología, 9, 105-119.

MÉNDEZ MARTínEZ Roberto (2009), « Ópera y discurso histórico-narrativo en Concierto barroco de Alejo Carpentier », en ligne sur Gáspar, El Lugarreño : <www.ellugareno.com/2009/05/opera-ydiscurso-historico-narrativo-en.html> (23 mai 2019).

Meschonnic Henri (1973), Pour la poétique, II : Épistémologie de l'écriture. Poétique de la traduction, Paris : Gallimard.

Meschonnic Henri (1997), De la langue française : essai sur une clarté obscure, Paris : Hachette.

MESCHONNIC Henri (1999), Poétique du traduire, Lagrasse : Verdier.

Mimoso-Ruiz Duarte (1980), «Vision de la musique au XVIII ${ }^{\mathrm{e}}$ siècle : Concert baroque de Alejo Carpentier (1976) », Études sur le XVIII siècle, Strasbourg : Faculté des lettres modernes, 115-135.

MonTES Cristián (1998), « Música e identidad en la novela Concierto barroco de Alejo Carpentier », Revista de Humanidades, 3, 7-18.

Montoya CAMPUZANo Pablo (2001), La musique dans l'œuvre d'Alejo Carpentier (thèse de doctorat), Université Sorbonne Nouvelle - Paris 3, Paris.

MORELL Hortensia R. (1983), « Contextos musicales en Concierto barroco », Revista iberoamericana, XLIX(123-124), 335-350.

Moulin CIVIL Françoise (2012), « La mise en scène de l'histoire : La aprendiz de bruja (1956) d'Alejo Carpentier », F. Parisot (dir.), Alejo Carpentier à l'aube du XXI siècle, Paris : L'Harmattan, 95-105.

MÜLLER-BERGH Klaus (1975), « Sentido y color de Concierto barroco », Revista Iberoamericana, XLVI(92-93), 445-464

OSEKI-DÉPRÉ Inês (2007), De Walter Benjamin à nos jours : essais de traductologie, Paris : H. Champion. 
PARISOT Fabrice (2006), «L'intertextualité dans Concert baroque d'Alejo Carpentier : une mosaïque d'esthétiques variées », Cahiers de narratologie, 13, <http://narratologie.revues.org/367> (11 février 2019).

PAz Octavio (2004), El Laberinto de la soledad [1950]. Postdata. Vuelta al laberinto de la soledad, Mexico : Fondo de cultura económica.

Pelegrín Benito (1986), « Música literaria y música literal en Concierto Barroco de Alejo

Carpentier », Imán, 3, 200-222.

Pelegrín Benito (2003), Alejo Carpentier, écrire, décrire l'Amérique : partage des eaux et des cultures, Paris : Ellipses.

REYES Alfonso (2009), La experiencia literaria y otros ensayos [1942], Madrid : Fundación Banco Santander.

RoA Bastos Augusto (1977), Moi, le Suprême (A. Berman, trad.), Paris : Belfond.

RUBIO NAVARRo Gabriel María (1999), Música y escritura en Alejo Carpentier, Alicante : Universidad de Alicante.

SUCHET Myriam (2014), L'imaginaire hétérolingue : ce que nous apprennent les textes à la croisée des langues, Paris : Classiques Garnier.

VÁsquez Carmen (1991), « Moctezuma: de la ópera a la obra de Carpentier », J. Covo (dir.), La construction du personnage historique : aires hispaniques et hispano-américaines, Villeneuve-d'Ascq : Presses universitaires de Lille, 129-135.

VEGA José Luis (1981), « Tiempo ritmo e historia en “Concierto Barroco" », Sin Nombre, LXI(2), 67-80.

VICKERS David (2006), « L'exhumation d'un trésor. La redécouverte de Motezuma », Vivaldi, Motezuma, Hambourg : Deutsche Grammophon, 48-52.

\section{NOTES}

1. Pour une bibliographie sur cette question, voir par exemple Rubio Navarro (1999 : 240-244).

2. Parmi les études consacrées à la musique dans Concierto barroco, on peut notamment citer Müller-Bergh (1975), Mimoso-Ruiz (1980), Vega (1981), Morell (1983), Pelegrín (1986), Vásquez (1991), Montes (1998), Méndez Martínez (2009).

3. Comme l'indique Müller-Bergh (1975: 445) et le rappelle Parisot (2006: § 1), Concierto barroco constituait pour Carpentier « une Somme théologique de [son] art dans la mesure où [ce livre] contient tous les mécanismes du baroque simultanément ".

4. Voir Suchet (2014: en particulier 217-264 sur les rapports entre «textes hétérolingues » et traduction).

5. Le Maître, après avoir été témoin de la répétition générale du Motezuma de Vivaldi, s'exclame : « ¿Y dónde metieron a Doña Marina, en toda esta mojiganga mexicana?» (Carpentier, 1998:75) Traduction de René L.F. Durand: «Et où a-t-on fourré Doña Marina dans cette mascarade mexicaine ?» (Carpentier, $1976: 92$ et $1991: 157)$

6. Observons que Carpentier aura seulement pu prendre connaissance du livret de Giusti, la partition de Vivaldi n'ayant été (partiellement) retrouvée qu'après sa mort par le musicologue allemand Steffen Voss en 2002 (Vickers, 2006 : 48 ; Méndez Martínez, 2009).

7. Lettre de Carpentier à Arnaldo Orfila Reynal datant de 1974, l'année même de la publication de Concierto barroco (citée par Parisot, 2006, § 2). Voir, à ce propos, Müller-Bergh (1975 : 462) : « Tout 
comme dans d'autres œuvres (notamment El reino de este mundo et El siglo de las luces), le romancier cubain construit son récit en se fondant sur des textes précis et documentés. Selon moi, Concierto barroco est peut-être le roman le plus "textuel" de Carpentier. » (Notre traduction) 8. Dans ce mouvement, André Breton et le surréalisme pourraient constituer un contre-exemple qui fait d'ailleurs l'objet des critiques de Carpentier (voir le prologue de El reino de este mundo et sa reprise dans l'essai intitulé De lo real maravilloso americano dans Carpentier, 1984 : 68-79).

9. Dans le prologue de son opéra, Giusti écrit: «Tout ce que j'abandonne de vrai, tout ce que j'ajoute de vraisemblable, je le fais pour m'adapter à la Scène et pour que cet opéra appelé MOTEZUMA soit représenté de la façon la moins imparfaite possible.» (Cité par Müller-Bergh, 1975 : 462 ; notre traduction.)

10. Pour une mise en perspective du rôle de Doña Marina en tant qu'interprète "témoin privilégié de l'histoire ", voir Delisle et Woodsworth (1995 : 259-260).

11. Cette solidarité entre les deux logiques peut se lire dès l'épisode de la Tour de Babel, «scène primitive de la théorie du langage, et de la traduction» (Meschonnic, 1999: 445). Pour une synthèse sur la mise en regard, par plusieurs penseurs modernes, de la logique historique et de la logique de la traduction, voir notamment De Launay (2006:52-72).

12. Sur la possibilité de telles analogies, voir Carpentier (1972:39-40).

13. Pour un relevé méthodique des exemples de transculturation dans Concierto barroco, voir Martín Ghioldi (2007).

14. Voir l'article «Las jitanjáforas » d'Alfonso Reyes (2009: 185-213) - article paru en 1942, qui a fait date et qui mentionne en incipit un recueil de poèmes de l'écrivain cubain Mariano Brull. Voir aussi Carpentier (1976: 292) et Montoya Campuzano (2001:53-55 et 188).

15. L'analyse de Cordonnier (1989), qui se réclame de la critique des traductions élaborée par Meschonnic, est très sévère. Pour une analyse plus équilibrée et spécifiquement consacrée à la traduction de Concierto barroco, nous renvoyons à un sous-chapitre de l'ouvrage de Malingret (2002 : 164-173) dans lequel l'auteur compare, selon la méthode de la critique descriptive de Gideon Toury, les éditions unilingue et bilingue réalisées par Durand.

16. La part de naïveté qui guette toujours de tels points de vue était relevée en 1973 par Michel Deguy qui évoque «tels écrivains habiles à tirer sur le fonds de mémoire de traditions non occidentales, qu'ils s'avisent soudain d'exploiter pour en rafraîchir la vieille Europe, nationalisant et acculturant les rythmes, les mythes, les fables et les formes de leurs "pays"; le fait que nous soyons rattrapés par ce passé devenant nôtre, en provenance de l'Afrique ou de l'Asie ou de l'Amérique du Sud, par tels poètes ambassadeurs, ce choc en retour qui nous revitalise un temps - mais qui est sans doute un effet de décision occidentale [...] n'y a-t-il pas là plutôt une régression partielle qu'une trouée d'avenir? [...] Oui, nous sommes condamnés à endurer et traverser le nihilisme, à traverser notre désert, "naufrage vers aucun îlot perdu" peut-être, comme disait Mallarmé dans un poème pourtant de jeunesse » (1973 : 122-123).

17. Voir Berman (2012: 271, pour la dette envers Meschonnic) et Meschonnic (1999: 97, pour une explicitation de la notion telle que Meschonnic l'entend). Il faut insister sur le fait que, dans ce schéma, la notion d'oralité ne doit pas être confondue avec le parlé qui seul s'oppose alors à l'écrit.

18. Malingret relève ainsi les traces de «tendances déformantes» dans les traductions de Concierto barroco par René L.F. Durand (Malingret, 2002: 169, pour l'ennoblissement, la clarification et la rationalisation; ibid.: 171, pour l'homogénéisation et l'effacement des superpositions de langues) tout en ne partageant ni l'horizon ni la méthode de la critique bermanienne des traductions.

19. Rappelons l'exception de L'apprentie sorcière, pièce traduite en espagnol par Carmen Vásquez, et qui tourne précisément autour du personnage de l'interprète Doña Marina.

20. Nous traduisons cette citation comme les citations ultérieures du volume Ensayos (Carpentier, 1984). 
21. Benito Pelegrín fait, concernant le style de Carpentier, l'observation suivante: «La prédilection de Carpentier va à un type de phrase latine, très différente de l'ordre habituel du français (sujet, verbe, complément) contre la rigidité de laquelle s'élevaient déjà La Bruyère, Fénelon et d'autres. » $(2003: 193)$

22. Remarquons que Carpentier cite en exemple Moi, le Suprême de Roa Bastos, une œuvre qu'Antoine Berman a choisi de traduire en français et qui lui sert également d'exemple pour développer ses propos sur « l'oralité populaire » (Berman, 1999 : 14).

23. Carpentier souligne ailleurs qu' « un profond fossé s'est creusé entre l'espagnol d'Amérique et l'espagnol d'Europe » $(1984: 131)$ et cette idée pourrait être, là encore, remise en perspective et contestée. Voir notamment Juan Goytisolo qui déclare en 1987 : «Cette division entre littérature espagnole, d'un côté, et littérature latino-américaine, de l'autre, me parait foncièrement fausse [...] il y a la même distance entre la littérature espagnole et celle de Cuba, celle de Cuba et celle de l'Argentine.» (Cité par Malingret, 2002 : 41) Voir aussi, plus généralement, les observations de Myriam Suchet (2014 : 43-50) concernant « la langue » et les phénomènes d'« appropriation » de la langue de l'autre en littérature.

24. Henri Meschonnic dans De la langue française: essai sur une clarté obscure (1997) propose une généalogie historique de ces tendances.

25. Dans ce même passage, à propos de Gargantua et de Pantagruel, Carpentier observe qu'il s'agit de «l'expression la plus complète, la plus savoureuse, de ce que pourrait offrir la plénitude de la langue française » (ibid.).

26. Voir notamment Berman (1979 : 165-166; $1980: 4 ; 2012: 72)$.

27. L'ouvrage de Bakhtine sur Rabelais est explicitement mentionné dans le compte rendu que fait Berman (1979 : 165) de l'édition française de América latina en su literatura. Il figure aussi dans la bibliographie de L'épreuve de l'étranger (1995a : 310).

28. Voir la citation de Pannwitz dans «La tâche du traducteur »: "Nos traductions même les meilleures partent d'un faux principe voulant germaniser le sanscrit le grec l'anglais au lieu de sanscritiser d'helléniser d'angliciser l'allemand. » (Benjamin, $2000: 260$ )

29. «En partie» car il nous semble possible, en effet, d'avancer que les œuvres de certains écrivains français actuels, notamment Michel Deguy, Pierre Guyotat ou Céline Minard, laissent voir les "potentialités linguistiques et culturelles » caractéristiques de "l'oralité » visée par Berman.

30. Pour le domaine latino-américain, il précisera ainsi que Borges ne répond pas, selon lui, à une telle caractérisation (Berman, $2008: 62$ ).

31. Nous nous permettons de renvoyer à A. Talbot, « Antoine Berman et la copia traductive : de la métaphore au concept » (à paraître).

32. À propos de Carpentier, Malingret évoque, dans le même ordre d'idée, «la joyeuse confusion qui peut naître des phrases longues et peu structurées de Carpentier» (2002:173). Voir aussi Pelegrín (2003 : 178 et 193-194).

33. «Écriture-de-traduction » qui serait « un mode d'écriture irréductible » (Berman, 1995a : 249) particulièrement sensible dans ces "ces "zones textuelles" où le traducteur a écrit-étranger en français » (Berman, 1995b : 66).

34. Berman remarque effectivement que si l'« écriture-de-traduction » est " un mode d'écriture irréductible », il n'en reste pas moins que «dans toute écriture littéraire, il y a toujours trace d'un tel rapport » (1995a : 249).

35. Cette "scène" est figurée, à travers la description ironique d'un tableau, dès le premier chapitre de Concierto barroco (Carpentier, 1998 : 11-12). Sur la position ambiguë de Doña Marina dans la pièce L'apprentie sorcière, voir Moulin Civil (2012 : 102-105) qui évoque une «rencontre littéralement inaugurale » (ibid. : 105).

36. Henri Meschonnic, lequel joue peut-être ici encore le rôle de "compagnon secret» de Berman, observe qu'«une seule idée-force fait l'unité de [l']œuvre [de Bakhtine], jusqu'au 
ressassement, et le chevauchevement d'un livre sur l'autre : la conception carnavalesque du monde » (1973: 191).

\section{RÉSUMÉS}

Le présent article est consacré au roman Concierto barroco d'Alejo Carpentier. À travers l'analyse de quelques motifs musicaux, il s'agit de manifester deux logiques à l'œuvre dans ce texte : la logique historique et la logique de la traduction. En faisant dialoguer des remarques critiques de Carpentier et des réflexions du traducteur et traductologue Antoine Berman, l'objectif est de montrer comment le "défi » représenté par la traduction de ce roman en français, comme d'autres œuvres littéraires latino-américaines, peut être rapporté à un « desencuentro linguistique et culturel » qui fait écho à la scène inaugurale au cœur de l'intrigue de Concierto barroco, à savoir le face-à-face entre Cortés et Montezuma. Le bref roman de Carpentier devient par là même une invitation à repenser la traduction dans sa solidarité avec la logique historique pour rendre justice à la créolité ou au polymétissage qui innervent ce texte ainsi que d'autres romans latinoaméricains.

The present paper uses an analysis of some of the musical motifs in Alejo Carpentier's novel Concierto barroco to explore two of the logics at play in this text: the historical logic and the translation logic. By setting Carpentier's critical remarks against the reflections of the translator and translation researcher Antoine Berman, the objective is to show how the "challenge" of translating this book, like other Latin-American novels, into French can be likened to a "linguistic and cultural desencuentro" that echoes the inaugural scene at the center of Concierto barroco's story, that is, the face-off between Cortez and Montezuma. Viewed in this way, Carpentier's short novel highlights the importance of reappraising the relationship between translation and history in order to give justice to the multi-ethnic mixing that inflames both this book and many other Latin-American novels.

\section{INDEX}

Mots-clés : Alejo Carpentier, Antoine Berman, traduction, polymétissage

Keywords : Alejo Carpentier, Antoine Berman, translation, multi-ethnic mixing

\section{AUTEUR}

\section{AURÉLIEN TALBOT}

Université Grenoble Alpes, ILCEA4 